\title{
Two-sided Matching Decision with Two-granularity Uncertain and Incomplete Linguistic Terms
}

\author{
Yue Qi \\ School of Information Management, Jiangxi University of Finance and Economics, \\ Nanchang, China \\ yueqichina@126.com
}

\begin{abstract}
With respect to the two-sided matching problem, where the agents' preferences are in the format of two-granularity uncertain and incomplete linguistic terms, a matching method is presented. Some definitions on uncertain linguistic term and 2-tuple are firstly given. The two-sided matching problem with two-granularity uncertain and incomplete linguistic terms is described. Then, uncertain and incomplete linguistic term matrixes are transformed into 2tuple matrixes. Furthermore, a multi-objective optimization model is developed by using the extended 2-tuple weighted average. By using the 2-tuple arithmetic mean, the normalization method and the linear weighted method, the multi-objective optimization model can be converted into a single-objective optimization model. By solving the optimization model, the matching alternative can be obtained. A matching example illustrates the feasibility and effectiveness of this method.
\end{abstract}

Keywords: two-sided matching, two-granularity, uncertain and incomplete linguistic term, 2-tuple, optimization model

\section{Introduction}

There are plenty of two-sided matching problems in many fields of real life, such as marriage assignment [1-4], college admission [5-8], employee selection [9-12], and CEOs to companies [13, 14], Therefore two-sided matching is a research topic with extensive application backgrounds.

At present, the two-sided matching problems with different forms of information have attracted extensively attentions. For example, Gale and Shapley initially investigate the concept, existence, optimality and algorithm of stable matchings [15]. The notions of vonNeumann-Morgenstern stable sets are adopted to determine which matchings are possibly stable when agents are farsighted [16]. Boon and Sierksma match position with player in soccer team formation using linear optimization models [17]. Sethuraman, et al., focus on the geometric structure of fractional stable matchings in the stable admission problem [18]. Uetake and Watanabe propose an approach to estimate a non-transferable utility in two-sided matching models [19].

The existing studies develop the matching methods, and expand the actual application background. However, due to the complexity of actual problems, the ambiguity of thinking judgement, the preferences provided by agents are in the format of uncertain and incomplete linguistic terms, and the granularity of the linguistic term set on two sides is also different. The existing studies seldom consider this case. Therefore, how to consider the two-sided matching problem with two-granularity uncertain and incomplete linguistic terms is a valuable research topic. In this paper, a novel two-sided matching method is presented. 
The remainder of this paper is arranged as follows: Section 2 introduces some concepts of uncertain linguistic term and 2-tuple. Section 3 formulates the two-sided matching problem with two-granularity uncertain and incomplete linguistic terms. Section 4 proposes a new method. Section 5 gives a matching example. Some conclusions are given in Section 6.

\section{Some Definitions}

Definition 1. Let $S=\left\{s_{0}, s_{1}, \cdots, s_{l}\right\}$ be the set of linguistic terms. An uncertain linguistic term $\hat{s}$ is expressed in $\hat{s}=\left\{s^{L}, s^{L+1}, \cdots, s^{U}\right\}$, where $s^{L}, s^{L+1}, \cdots, s^{U} \in S, L \leq U$. Specially, if $s^{L}=s^{U}$, then $\bar{s}$ reduces to a linguistic term. For simplicity, we express $\bar{s}$ as $\hat{s}=\left[s^{L}, s^{U}\right]$.

Remark 1. In the two-sided matching problem, if $\hat{s}=\left[s^{L}, s^{U}\right]$ is an uncertain linguistic term, then the occurrence possibility of linguistic terms $s^{L}, \ldots$, and $s^{U}$ are the same.

Definition 2. Let $\hat{r}=\left[r^{L}, r^{U}\right]$ be an uncertain linguistic term, then the probability vector on $\hat{r}=\left[r^{L}, r^{U}\right]$ is expressed in $\hat{p}_{\hat{r}}=\left(p_{r}^{L}, \cdots, p_{r}^{k}, \cdots, p_{r}^{U}\right)$, where $p_{\dot{r}}^{k}=1 /(U-L+1)$.

Definition 3. Let $\hat{r}=\left[r^{L}, r^{U}\right]$ be an uncertain linguistic term, and $\hat{p}_{\hat{r}}$ be the probability vector on $\hat{r}$, then by Definitions 2 and 3, the expectation of $\hat{r}$ (noted as $E(\hat{r}))$ is calculated by

$$
E(\hat{r})=\theta\left(\sum_{k=r^{r}}^{r^{v}} \frac{\theta^{-1}(k, 0)}{U-L+1}\right)
$$

where $\theta$ is a symbolic translation, and $\theta^{-1}$ is the inverse function of $\theta$.

In order to deal with 2-tuples, the extended 2-tuple weighted average is give as follows according to references [20,21].

Definition 4. Let $x=\left\{\left(r_{1}, \alpha_{1}\right),\left(r_{2}, \alpha_{2}\right), \cdots,\left(r_{f}, \alpha_{f}\right)\right\}$ be a set of 2-tuples and $w=\left\{w_{1}, w_{2}, \cdots, w_{f}\right\}$ be the associated weights $\left(w_{i} \in[0,1], \sum w_{i} \in[0,1]\right)$. The extended 2-tuple weighted average is defined as

$$
\tilde{\bar{r}}^{w}=\left\{\begin{array}{l}
\left\{\begin{array}{c}
\left(\sum_{c=1}^{f} \theta^{-1}\left(r_{c}, \alpha_{c}\right) w_{c}\right) \\
\theta \\
\mid \sum_{c=1}^{f} w_{c}
\end{array}\right\}, \quad 0<\sum_{c=1}^{f} w_{c} \leq 1 \\
\theta\left(\sum_{c=1}^{f} \theta^{-1}\left(r_{c}, \alpha_{c}\right) w_{c}\right)=\theta(0), \quad \sum_{c=1}^{f} w_{c}=0
\end{array}\right.
$$

\section{The Problem}

This paper considers the two-sided matching problem with two-granularity uncertain and incomplete linguistic terms. The notation of the considered two-sided matching problem is provided as follows.

$P=\left\{P_{1}, P_{2}, \cdots, P_{m}\right\}$ : the set of agents of side $P, m \geq 2 ;$

$P_{i}:$ the $i$ th agent of side $P$;

$Q=\left\{Q_{1}, Q_{2}, \cdots, Q_{n}\right\}:$ the set of agents of side $Q, n \geq m ;$ 
$Q_{j}$; the $j$ th agent of side $Q$;

$S_{P}=\left\{s_{0}^{P}, s_{1}^{P}, \cdots, s_{p}^{P}, \phi\right\}:$ the extended set of satisfaction linguistic terms of side $P$;

$p+2:$ the cardinality of set $S_{P}$;

$U_{P}=\left[\hat{u}_{i j}^{P}\right]_{m \times n}$ : the satisfaction matrix from side $P$ to $Q$,

$\bar{u}_{i j}^{P}=\left[u_{i j}^{P L}, u_{i j}^{P R}\right]:$ the uncertain and incomplete linguistic term for agent $P_{i}$ with respect to agent $Q_{j}, u_{i j}^{P L}, u_{i j}^{P R} \in S_{P}, u_{i j}^{P L} \leq u_{i j}^{P R}$

$S_{Q}=\left\{s_{0}^{Q}, s_{1}^{Q}, \cdots, s_{q}^{Q}\right\}$ : the extended set of satisfaction linguistic terms of side $Q$;

$q+2:$ the cardinality of set $S_{Q}$;

$U_{Q}=\left[\hat{u}_{i j}^{Q}\right]_{m \times n}$ : the satisfaction uncertain linguistic term matrix from side $Q$ to $P$;

$\bar{u}_{i j}^{Q}=\left[u_{i j}^{Q L}, u_{i j}^{Q R}\right]:$ the uncertain and incomplete linguistic term for agent $Q_{j}$ with respect to agent $P_{i}, u_{i j}^{Q L}, u_{i j}^{Q R} \in S_{Q}, u_{i j}^{Q L} \leq u_{i j}^{Q R}$;

$\mu_{T}$ : the set of matching pairs, $\mu_{T}=\left\{\left(A_{i}, B_{\sigma(i)}\right) \mid i=1, \cdots, m\right\}$;

$\sigma(1), \cdots, \sigma(m)$ : the permutation of $1,2, \cdots, m$;

$\mu_{o}$ : the set of single pairs, $\mu_{o}=\left\{\left(B_{j}, B_{j}\right) \mid j \in\{1, \cdots, n\} \backslash\{\sigma(1), \cdots, \sigma(m)\}\right\}$;

$\mu$ : the two-sided matching or matching alternative, $\mu=\mu_{T} \cup \mu_{o}$.

The problem is how to obtain the reasonable matching alternative based on two-granularity uncertain and incomplete linguistic term matrixes $U_{P}=\left[\hat{u}_{i j}^{P}\right]_{m \times n}$ and $U_{Q}=\left[\hat{u}_{i j}^{Q}\right]_{m \times n}$.

\section{The Proposed Method}

Firstly, by Eq. (1), uncertain and incomplete linguistic term matrixes $U_{P}=\left[\hat{\bar{u}}_{i j}^{P}\right]_{m \times n}$ and $U_{Q}=\left[\hat{u}_{i j}^{Q}\right]_{m \times n}$ are transformed into 2-tuple matrixes $T_{P}=\left[\left(u_{i j}^{P}, \alpha_{i j}^{P}\right)\right]_{m \times n}$ and $T_{Q}=\left[\left(u_{i j}^{Q}, \alpha_{i j}^{Q}\right)\right]_{m \times n}$, where

$$
\begin{aligned}
& \left(u_{i j}^{P}, \alpha_{i j}^{P}\right)=\theta\left(\frac{\theta^{-1}\left(u_{i j}^{P L}, 0\right)+\theta^{-1}\left(u_{i j}^{P R}, 0\right)}{2}\right) \\
& \left(u_{i j}^{Q}, \alpha_{i j}^{Q}\right)=\theta\left(\frac{\theta^{-1}\left(u_{i j}^{Q L}, 0\right)+\theta^{-1}\left(u_{i j}^{Q R}, 0\right)}{2}\right)
\end{aligned}
$$

Remark 2. In Eqs. (3) and (4), if $u_{i j}^{P L}=u_{i j}^{P R}=\phi$, then $\left(u_{i j}^{P}, \alpha_{i j}^{P}\right)=\phi$; if $u_{i j}^{Q L}=u_{i j}^{Q R}=\phi$, then $\left(u_{i j}^{Q}, \alpha_{i j}^{Q}\right)=\phi$.

Based on 2-tuple matrixes $T_{P}=\left[\left(u_{i j}^{P}, \alpha_{i j}^{P}\right)\right]_{m \times n}$ and $T_{Q}=\left[\left(u_{i j}^{Q}, \alpha_{i j}^{Q}\right)\right]_{m \times n}$, we consider to construct an optimization model under the matching constraints. On the one hand, according to the characteristics of linguistic term and 2-tuple, we know that the greater $\left(u_{i j}^{P}, \alpha_{i j}^{P}\right)$ or $\left(u_{i j}^{Q}, \alpha_{i j}^{Q}\right)$ is, the greater the satisfaction degree is. On the other hand, the matching constraints can be interpreted as $\sum_{j=1}^{n} x_{i j} \leq 1$ and $\sum_{i=1}^{m} x_{i j} \leq 1$, where $x_{i j}=\left\{\begin{array}{ll}1, & \mu\left(P_{i}\right)=Q_{j} \\ 0, & \mu\left(P_{i}\right) \neq Q_{j}\end{array}\right.$. Furthermore, by Eq. (2), the following multi-objective optimization model (5) can be established: 


$$
\begin{array}{ll}
\max & Z_{\left(P_{i}\right)}=\theta\left(\sum_{j=1}^{n} \theta^{-1}\left(u_{i j}^{P}, \alpha_{i j}^{P}\right) x_{i j} / \sum_{j=1}^{n} x_{i j}\right)=\theta\left(\sum_{j=1}^{n} \theta^{-1}\left(u_{i j}^{P}, \alpha_{i j}^{P}\right) x_{i j}\right), i=1,2, \cdots, m \\
\max & Z_{\left(Q_{j}\right)}=\theta\left(\sum_{i=1}^{m} \theta^{-1}\left(u_{i j}^{Q}, \alpha_{i j}^{Q}\right) x_{i j} / \sum_{i=1}^{m} x_{i j}\right)=\theta\left(\sum_{i=1}^{m} \theta^{-1}\left(u_{i j}^{Q}, \alpha_{i j}^{Q}\right) x_{i j}\right), j=1,2, \cdots, n \\
\text { s.t. } \quad & \sum_{j=1}^{n} x_{i j} \leq 1, i=1,2, \cdots, m \\
& \sum_{i=1}^{m} x_{i j} \leq 1, j=1,2, \cdots, n \\
& x_{i j} \in\{0,1\}, i=1,2, \cdots, m, j=1,2, \cdots, n
\end{array}
$$

Remark 3. In Eqs. (5a) and (5b), if $\left(u_{i j}^{P}, \alpha_{i j}^{P}\right)=\phi$, then $\theta\left(\sum_{j=1}^{n} \theta^{-1}\left(u_{i j}^{P}, \alpha_{i j}^{P}\right) x_{i j}\right)=\phi$; if $\left(u_{i j}^{Q}, \alpha_{i j}^{Q}\right)=\phi$, then $\theta\left(\sum_{i=1}^{m} \theta^{-1}\left(u_{i j}^{Q}, \alpha_{i j}^{Q}\right) x_{i j}\right)=\phi$.

Usually, the status of each agent of one side is the same. So, each agent of one side processes equal priority. Then the 2-tuple arithmetic mean $[20,21]$ is used. Hence, model (5) can be transformed into the following bi-objective optimization model (6):

$$
\begin{array}{ll}
\max & Z_{(P)}=\theta\left(\frac{1}{m} \sum_{i=1}^{m} \sum_{j=1}^{n} \theta^{-1}\left(u_{i j}^{P}, \alpha_{i j}^{P}\right) x_{i j}\right) \\
\max \quad Z_{(Q)}=\theta\left(\frac{1}{n} \sum_{j=1}^{n} \sum_{i=1}^{m} \theta^{-1}\left(u_{i j}^{Q}, \alpha_{i j}^{Q}\right) x_{i j}\right) \\
\text { s.t. } \quad \sum_{j=1}^{n} x_{i j} \leq 1, i=1,2, \cdots, m \\
& \sum_{i=1}^{m} x_{i j} \leq 1, j=1,2, \cdots, n \\
& x_{i j} \in\{0,1\}, i=1,2, \cdots, m, j=1,2, \cdots, n
\end{array}
$$

Considering the characteristics of symbolic translation $\theta$, the optimal solution of the following optimization model (7) is that of model (6):

$$
\begin{array}{ll}
\max & Z_{(P)}=\frac{1}{m} \sum_{i=1}^{m} \sum_{j=1}^{n} \theta^{-1}\left(u_{i j}^{P}, \alpha_{i j}^{P}\right) x_{i j} \\
\max & Z_{(Q)}=\frac{1}{n} \sum_{j=1}^{n} \sum_{i=1}^{m} \theta^{-1}\left(u_{i j}^{Q}, \alpha_{i j}^{Q}\right) x_{i j} \\
\text { s.t. } \quad & \sum_{j=1}^{n} x_{i j} \leq 1, i=1,2, \cdots, m \\
& \sum_{i=1}^{m} x_{i j} \leq 1, j=1,2, \cdots, n \\
& x_{i j} \in\{0,1\}, i=1,2, \cdots, m, j=1,2, \cdots, n
\end{array}
$$

To solve model (7), due to $p \neq q$, Eqs. (7a) and (7b) should be normalized firstly. Let $w_{P}$ and $w_{Q}$ be the weight of $Z_{(P)}$ and $Z_{(Q)}$, such that $w_{P}, w_{Q} \in[0,1], w_{P}+w_{Q}=1$, then model (7) can be transformed into the following single-objective optimization model (8): 


$$
\begin{array}{ll}
\max & Z=\frac{1}{m n} \sum_{i=1}^{m} \sum_{j=1}^{n}\left(w_{P} \theta^{-1}\left(u_{i j}^{P}, \alpha_{i j}^{P}\right)+w_{Q} \theta^{-1}\left(u_{i j}^{Q}, \alpha_{i j}^{Q}\right)\right) x_{i j} \\
\text { s.t. } & \sum_{j=1}^{n} x_{i j} \leq 1, i=1,2, \cdots, m \\
& \sum_{i=1}^{m} x_{i j} \leq 1, j=1,2, \cdots, n \\
& x_{i j} \in\{0,1\}, i=1,2, \cdots, m, j=1,2, \cdots, n
\end{array}
$$

Model (8) can be solved by the existing mathematical optimization software. Based on the obtained optimal solution, the matching alternative can be obtained.

In sum, an algorithm is developed and its step is displayed as follows:

Step 1. Transform uncertain and incomplete linguistic term matrixes $U_{P}=\left[\hat{u}_{i j}^{P}\right]_{m \times n}$ and $U_{Q}=\left[\hat{u}_{i j}^{Q}\right]_{m \times n}$ into 2-tuple matrixes $T_{P}=\left[\left(u_{i j}^{P}, \alpha_{i j}^{P}\right)\right]_{m \times n}$ and $T_{Q}=\left[\left(u_{i j}^{Q}, \alpha_{i j}^{Q}\right)\right]_{m \times n}$ by Eqs. (1), (3) and (4).

Step 2. Built the multiple-objective optimization model (5) based on 2-tuple matrixes $T_{P}=\left[\left(u_{i j}^{P}, \alpha_{i j}^{P}\right)\right]_{m \times n}$ and $T_{Q}=\left[\left(u_{i j}^{Q}, \alpha_{i j}^{Q}\right)\right]_{m \times n}$ by Eq. (2).

Step 3. Transform model (5) into model (6) by using the 2-tuple arithmetic mean.

Step 4. Transform model (6) into model (8) by using the normalization method and the linear weighted method.

Step 5. Obtain the matching alternative by solving model (8).

\section{Example}

The energy and environment trading service intermediary in Changsha plans to make energy matching between demanders (such as, industry manufacturers) and suppliers (such as, power companies). Four demanders $\mathrm{A}_{1}, \mathrm{~A}_{2}, \ldots, \mathrm{A}_{4}$ and six suppliers $\mathrm{B}_{1}, \mathrm{~B}_{2}, \ldots, \mathrm{B}_{6}$ participate in the matching process. Energy demander $A_{i}$ evaluates suppliers from market prospect, complexity, and price according to the satisfaction linguistic term set $S_{P}=\left\{s_{0}=\mathrm{VL}(\right.$ Very low $), s_{1}=\mathrm{L}(\mathrm{Low}), s_{2}=\mathrm{ML}($ Medium low $), s_{3}=\mathrm{M}($ Medium $)$, $s_{4}=\mathrm{MH}($ Medium high $), s_{5}=\mathrm{H}(\mathrm{High}), s_{6}=\mathrm{VH}($ Very high $\left.), \phi\right\}$. Supplier $B_{j}$ evaluates energy demanders from income, conversion speed, and technological level according to the satisfaction linguistic term set $s_{Q}=\left\{s_{0}=\mathrm{VL}\right.$ (Very low), $s_{1}=\mathrm{L}($ Low $), s_{2}=\mathrm{M}($ Medium $)$, $s_{3}=\mathrm{H}(\mathrm{High}), s_{4}=\mathrm{VH}($ Very high $\left.), \phi\right\}$. Uncertain and incomplete linguistic term matrixes $U_{P}=\left[\hat{u}_{i j}^{P}\right]_{4 \times 6}$ and $U_{Q}=\left[\hat{u}_{i j}^{Q}\right]_{4 \times 6}$ are given as follows.

$$
\begin{aligned}
U_{P}= & {\left[\begin{array}{cccccc}
{\left[s_{2}^{P}, s_{3}^{P}\right]} & {\left[s_{3}^{P}, s_{4}^{P}\right]} & {\left[s_{2}^{P}, s_{2}^{P}\right]} & {\left[s_{4}^{P}, s_{5}^{P}\right]} & {\left[s_{1}^{P}, s_{2}^{P}\right]} & \phi \\
{\left[s_{3}^{P}, s_{4}^{P}\right]} & \phi & {\left[s_{1}^{P}, s_{1}^{P}\right]} & {\left[s_{0}^{P}, s_{1}^{P}\right]} & {\left[s_{4}^{P}, s_{4}^{P}\right]} & {\left[s_{2}^{P}, s_{2}^{P}\right]} \\
{\left[s_{2}^{P}, s_{2}^{P}\right]} & {\left[s_{0}^{P}, s_{1}^{P}\right]} & {\left[s_{1}^{P}, s_{2}^{P}\right]} & \phi & {\left[s_{2}^{P}, s_{3}^{P}\right]} & {\left[s_{4}^{P}, s_{4}^{P}\right]} \\
{\left[s_{1}^{P}, s_{2}^{P}\right]} & \phi & {\left[s_{3}^{P}, s_{4}^{P}\right]} & {\left[s_{0}^{P}, s_{1}^{P}\right]} & {\left[s_{2}^{P}, s_{3}^{P}\right]} & {\left[s_{2}^{P}, s_{2}^{P}\right]}
\end{array}\right] } \\
U_{Q}= & {\left[\begin{array}{cccccc}
{\left[s_{2}^{Q}, s_{3}^{Q}\right]} & {\left[s_{3}^{Q}, s_{4}^{Q}\right]} & {\left[s_{2}^{Q}, s_{2}^{Q}\right]} & {\left[s_{3}^{Q}, s_{3}^{Q}\right]} & {\left[s_{1}^{Q}, s_{1}^{Q}\right]} & {\left[s_{0}^{Q}, s_{1}^{Q}\right]} \\
\phi & {\left[s_{2}^{Q}, s_{3}^{Q}\right]} & {\left[s_{1}^{Q}, s_{1}^{Q}\right]} & {\left[s_{2}^{Q}, s_{3}^{Q}\right]} & {\left[s_{1}^{Q}, s_{2}^{Q}\right]} & {\left[s_{2}^{Q}, s_{2}^{Q}\right]} \\
{\left[s_{2}^{Q}, s_{2}^{Q}\right]} & {\left[s_{0}^{Q}, s_{1}^{Q}\right]} & {\left[s_{2}^{Q}, s_{3}^{Q}\right]} & {\left[s_{1}^{Q}, s_{2}^{Q}\right]} & {\left[s_{0}^{Q}, s_{1}^{Q}\right]} & \phi \\
{\left[s_{1}^{Q}, s_{2}^{Q}\right]} & {\left[s_{2}^{Q}, s_{2}^{Q}\right]} & \phi & \phi & {\left[s_{2}^{Q}, s_{2}^{Q}\right]} & {\left[s_{1}^{Q}, s_{2}^{Q}\right]}
\end{array}\right] }
\end{aligned}
$$


To determine the reasonable matching alternative, a brief description of the matching process is given below.

Firstly, by Eqs. (1), (3) and (4), uncertain and incomplete linguistic term matrixes $U_{P}=\left[\hat{u}_{i j}^{P}\right]_{4 \times 6}$ and $U_{Q}=\left[\hat{u}_{i j}^{Q}\right]_{4 \times 6}$ are transformed into 2-tuple matrixes $T_{P}$ and $T_{Q}$ as follows.

$$
\begin{aligned}
& T_{P}=\left[\begin{array}{cccccc}
\left(s_{3}^{P},-0.5\right) & \left(s_{4}^{P},-0.5\right) & \left(s_{2}^{P}, 0\right) & \left(s_{5}^{P},-0.5\right) & \left(s_{2}^{P},-0.5\right) & \phi \\
\left(s_{4}^{P},-0.5\right) & \phi & \left(s_{1}^{P}, 0\right) & \left(s_{1}^{P},-0.5\right) & \left(s_{4}^{P}, 0\right) & \left(s_{2}^{P}, 0\right) \\
\left(s_{2}^{P}, 0\right) & \left(s_{1}^{P},-0.5\right) & \left(s_{2}^{P},-0.5\right) & \phi & \left(s_{3}^{P},-0.5\right) & \left(s_{4}^{P}, 0\right) \\
\left(s_{2}^{P},-0.5\right) & \phi & \left(s_{4}^{P},-0.5\right) & \left(s_{1}^{P},-0.5\right) & \left(s_{3}^{P},-0.5\right) & \left(s_{2}^{P}, 0\right)
\end{array}\right] \\
& T_{Q}=\left[\begin{array}{cccccc}
\left(s_{3}^{Q},-0.5\right) & \left(s_{4}^{Q},-0.5\right) & \left(s_{2}^{Q}, 0\right) & \left(s_{3}^{Q}, 0\right) & \left(s_{1}^{Q}, 0\right) & \left(s_{1}^{Q},-0.5\right) \\
\phi & \left(s_{3}^{Q},-0.5\right) & \left(s_{1}^{Q}, 0\right) & \left(s_{3}^{Q},-0.5\right) & \left(s_{2}^{Q},-0.5\right) & \left(s_{2}^{Q}, 0\right) \\
\left(s_{2}^{Q}, 0\right) & \left(s_{1}^{Q},-0.5\right) & \left(s_{3}^{Q},-0.5\right) & \left(s_{2}^{Q},-0.5\right) & \left(s_{1}^{Q},-0.5\right) & \phi \\
\left(s_{2}^{Q},-0.5\right) & \left(s_{2}^{Q}, 0\right) & \phi & \phi & \left(s_{2}^{Q}, 0\right) & \left(s_{2}^{Q},-0.5\right)
\end{array}\right]
\end{aligned}
$$

Then by Eq. (2), the multiple-objective optimization model (5) can be established based on 2tuple matrixes $T_{P}$ and $T_{Q}$. By using the 2-tuple arithmetic mean, model (5) is transformed into model (6). Furthermore, suppose $w_{A}=0.6$ and $w_{B}=0.4$, by using the normalization method and the linear weighted method, model (6) is transformed into model (8), i.e.,

$$
\begin{array}{ll}
\max & Z=\frac{1}{24} \sum_{i=1}^{4} \sum_{j=1}^{6}\left(0.6 \theta^{-1}\left(u_{i j}^{P}, \alpha_{i j}^{P}\right)+0.4 \theta^{-1}\left(u_{i j}^{Q}, \alpha_{i j}^{Q}\right)\right) x_{i j} \\
\text { s.t. } & \sum_{j=1}^{n} x_{i j} \leq 1, i=1,2, \cdots, 4 \\
& \sum_{i=1}^{m} x_{i j} \leq 1, j=1,2, \cdots, 6 \\
& x_{i j} \in\{0,1\}, i=1,2, \cdots, 4, j=1,2, \cdots, 6
\end{array}
$$

where coefficient matrix $C=\left[\left(0.6 \theta^{-1}\left(u_{i j}^{P}, \alpha_{i j}^{P}\right)+0.4 \theta^{-1}\left(u_{i j}^{Q}, \alpha_{i j}^{Q}\right)\right) / 24\right]_{4 \times 6}$ is shown in Table 1.

Table 1. Coefficient Matrix $C$

\begin{tabular}{ccccccc}
\hline$c_{i j}$ & $\mathrm{~B}_{1}$ & $\mathrm{~B}_{2}$ & $\mathrm{~B}_{3}$ & $\mathrm{~B}_{4}$ & $\mathrm{~B}_{5}$ & $\mathrm{~B}_{6}$ \\
\hline $\mathrm{A}_{1}$ & 0.0869 & 0.1217 & 0.0695 & 0.1364 & 0.0544 & $\phi$ \\
$\mathrm{A}_{2}$ & $\phi$ & $\phi$ & 0.0348 & 0.044 & 0.119 & 0.0873 \\
$\mathrm{~A}_{3}$ & 0.0867 & 0.0217 & 0.0783 & $\phi$ & 0.0861 & $\phi$ \\
$\mathrm{A}_{4}$ & 0.065 & $\phi$ & $\phi$ & $\phi$ & 0.1194 & 0.0933 \\
\hline
\end{tabular}

Finally, the matching alternative $\mu=\mu_{T}^{*} \cup \mu_{o}^{*}$ can be obtained by solving model (8), where $\mu_{T}^{*}=\left\{\left(P_{1}, Q_{4}\right),\left(P_{2}, Q_{5}\right),\left(P_{3}, Q_{1}\right),\left(P_{4}, Q_{6}\right)\right\}, \mu_{o}^{*}=\left\{\left(Q_{2}, Q_{2}\right),\left(Q_{3}, Q_{3}\right)\right\}$. Hence, $P_{1}$ matches with $Q_{4}, P_{2}$ matches with $Q_{5}, P_{3}$ matches with $Q_{1}, P_{4}$ matches with $Q_{6}, Q_{2}$ and $Q_{3}$ are unmatched.

\section{Conclusion}

The two-sided matching problem with two-granularity uncertain and incomplete linguistic terms is a hot topic with extensive application backgrounds. A matching method for solving 
the considered two-sided matching problem is proposed. Comparing with the existing methods, the proposed method has distinct characteristics as discussed below.

Firstly, the related concepts of uncertain linguistic term and 2-tuple are given. It is a beneficial supplement of theory of linguistic term. Secondly, a multi-objective optimization model is constructed. By solving it, it could be easier to obtain the reasonable stable matching alternative. Finally, the proposed method is theoretically sound and computationally simple and can be adopted for practical use.

\section{Acknowledgment}

This work was partly supported by the National Natural Science Foundation of China (Project Nos. 71261007, 71261006), the Humanities and Social Science Foundation of the Ministry of Education of China (Project No. 12YJC630080), Natural Science Fund of Jiangxi Province (Project No. 20132BAB201015), and Science and Technology Research Project of the Department of Education of Jiangxi Province (Project No. GJJ13292).

\section{References}

[1] R. W. Irving, D. F. Manlove and G. O’Malley,” Journal of Discrete Algorithms”, vol. 7, no. 213 (2009).

[2] C. C. Huang and T. Kavitha, Inform. Comput, vol. 222, no.180, (2013).

[3] F. Tamás, R.W. Irving and D. F. Manlove, Theor. Compu. Sci. vol. 381, no. 162, (2007).

[4] S. Lauermann and G. Nöldeke, J. Econ. Theory, vol. 151, no. 163, (2014).

[5] J. Pais, Econ. Theory, vol. 35, no. 99, (2008).

[6] P. Courty and M. Pagliero, Econ. Lett., vol. 108, no. 208, (2010).

[7] M. Koljatic, M. Silva and C. Rodrigo, Int. J. Educ. Dev., vol. 33, no. 106, (2013).

[8] W. C. Chen and Y. C. Kao, Econ. Lett., vol. 122, no. 296, (2014).

[9] K. J. Lauver and A. Kristof-Brown, J. Vocat. Behav., vol. 59, no. 454, (2001).

[10] R. Mendes, G. J. V. D. Berg and M. Lindeboom, "Labour Economics", vol. 17, no. 919, (2010).

[11] A. Golec and E. Kahya, Comput. Ind. Eng., vol. 52, no. 143, (2007).

[12] J. Zhou, Econ. Model, vol. 26, no. 1193, (2009).

[13] X. Gabaix and A. Landier, Q. J. Econ., vol. 123, no. 49 (2008).

[14] S. Allgood, K. A. Farrell, and R. Kamal, "Journal of Corporate Finance”, vol. 18, no. 1051, (2012).

[15] D. Gale, and L. Shapley, Am. Math. Mon. vol. 69, no. 9, (1962).

[16] Mauleon, V. J. Vannetelbosch, and W. Vergote, "Theoretical Economics", vol. 6, no. 499, (2011).

[17] H. Boon, and G. Sierksma, Eur. J. Oper. Res. 148, 277 (2003).

[18] J. Sethuraman, C. P. Teo, and L. Qian, Math. Oper. Res., vol. 31, no. 581, (2006).

[19] K. Uetake and Y. Watanabe, Econ. Lett., vol. 116, no. 535, (2012).

[20] F, Herrera and L. Martínez, IEEE T. Syst. Man. Cy. B. vol. 31, no. 227, (2001).

[21] F, Herrera, E, Herrera-Viedma and L. Martínez, "Fuzzy Sets and Systems", vol. 114, no. 43, (2000).

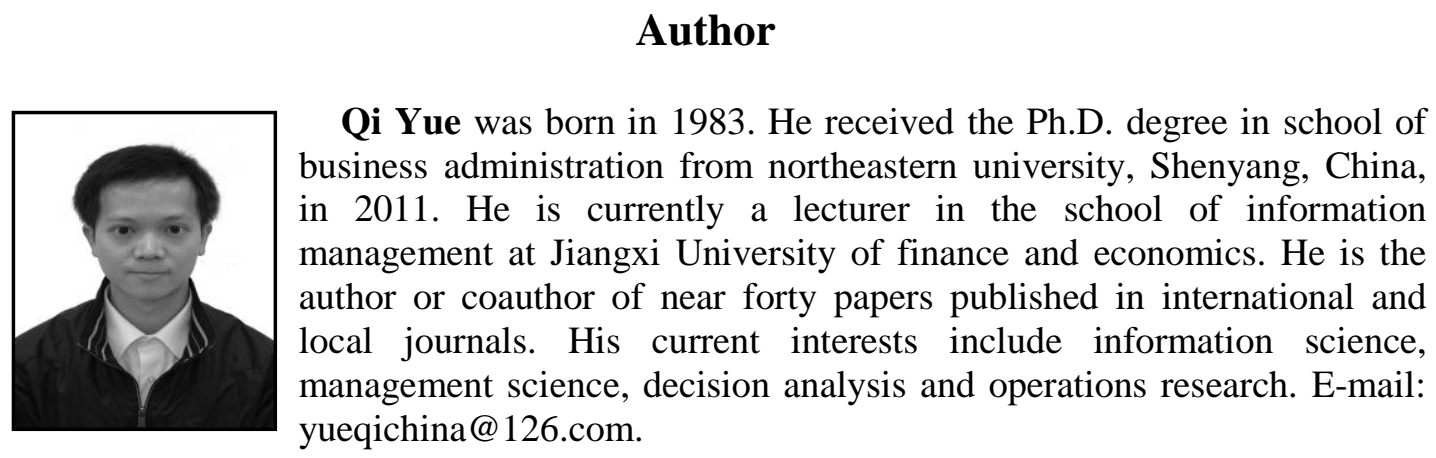


International Journal of Multimedia and Ubiquitous Engineering Vol. 10, No. 2 (2015) 